\title{
Protective effects of new Wenshen Shengjing Decoction on cyclosporine-induced impairment of testosterone synthesis and spermatogenic apoptosis
}

\author{
XIAOYAN PAN ${ }^{1}$, XIYAN WANG $^{2}$, XUENAN WANG $^{3}$, WANSHENG ZHANG $^{4}$, ZHANXUAN SUN $^{1}$, \\ XUANXUAN LIANG ${ }^{1}$, XUE ZHANG ${ }^{1}$, WENJUN LI ${ }^{1}$ and ZHIXIN LI ${ }^{1}$ \\ ${ }^{1}$ Department of Histology and Embryology; ${ }^{2}$ Medical Experimental Center, Jilin Medical University, Jilin, Jilin 132013; \\ ${ }^{3}$ Reproductive Medicine Center of The Affiliated Hospital of Jining Medical College, Jining, Shandong 272029; \\ ${ }^{4}$ Department of Urology, Affiliated Hospital of Jilin Medical University, Jilin, Jilin 132013, P.R. China
}

Received May 4, 2016; Accepted March 31, 2017

DOI: $10.3892 /$ etm.2017.5473

\begin{abstract}
The aim of the present study was to investigate the potential protective effects of new Wenshen Shengjing Decoction (new WSSJD; including Cornu Cervi Nippon Parvum, Panax ginseng, Cynomorium songaricum, Cistanche deserticola, Radix Astragali, Epimedium brevicornum and Angelica sinensis) on cyclosporine-induced impairment of testosterone synthesis and spermatogenic apoptosis in mice. A total of 90 adult male Kunming mice were divided into the following 6 groups: Control (no intervention), dimethylsulfoxide (DMSO; received only DMSO), cyclosporine A (CsA), clomifene citrate (CC; CsA + CC, $15 \mathrm{mg} / \mathrm{kg} /$ day), WSSJD (CsA + WSSJD, crude drug $12 \mathrm{~g} / \mathrm{kg} /$ day) and new WSSJD (CsA + new WSSJD, crude drug $12 \mathrm{~g} / \mathrm{kg} /$ day). All mice were treated for 30 days via oral gavage. The testes were subsequently fixed and stained with hematoxylin \& eosin to assess the development of seminiferous epithelia. Immunohistochemical techniques were used to detect the expression of luteinizing hormone receptor (LHR) and P450 side chain cleavage $(\mathrm{P} 450 \mathrm{scc})$ in testicular Leydig cells. In addition, the apoptosis of spermatogenic cells in the testes was detected using a terminal dexynucleotidyl transferase-mediated dUTP nick-end labeling assay, and flow cytometry was used to analyze the survival rate and early apoptosis of sperm in the epididymis. Compared with the CsA and CC groups, new WSSJD administration significantly increased levels of serum testosterone and the expressions of LHR and P450scc in testicular Leydig cells $(\mathrm{P}<0.05)$, while the apoptosis of spermatogenic cells in the seminiferous tubules and early apoptosis of mature sperm were significantly decreased $(\mathrm{P}<0.05)$. These
\end{abstract}

Correspondence to: Professor Zhixin Li, Department of Histology and Embryology, Jilin Medical University, 5 Jilin Street, Jilin, Jilin 132013, P.R. China

E-mail: pxy19790122@163.com

Key words: new Wenshen Shenjing Decoction, cyclosporine, testes, testosterone synthesis, cell apoptosis results suggest that new WSSJD may ameliorate CsA-induced spermatogenic damage in male mice by enhancing testosterone synthesis and the secretion of testicular Leydig cells, and by reducing the apoptosis of spermatogenic cells.

\section{Introduction}

Cyclosporine $\mathrm{A}(\mathrm{Cs} \mathrm{A})$ is a commonly used immunosuppressive agent that specifically targets $\mathrm{T}$ cells and inhibits the secretion of interleukin-2, and dramatically increases the success rate of liver, kidney and cornea transplantations, as well as the survival rate of recipients (1-3). However, immunosuppression induced by long-term CsA use significantly increases the risk of cardiovascular diseases, infection and malignant tumors (4). Eid et al (5) reported that the concentration and motility of sperm were negatively correlated with the concentration of serum CsA in male kidney transplant recipients, suggesting that CsA may induce dysfunction of the reproductive system in male recipients. Furthermore, it has been documented that CsA may be responsible for impaired spermatogenesis and damage to the male reproductive system (6).

Previous studies have demonstrated that CsA decreased the levels of serum testosterone by influencing testosterone biosynthesis and secretion in the testes. He et al (7) observed that rats treated with $40 \mathrm{mg} / \mathrm{kg}$ /day CsA exhibited significantly higher levels of luteinizing hormone (LH) and significantly lower levels of testosterone in the serum, leading to impaired testicular development. Ali et al (8) reported that treatment with CsA decreased the expression of $\mathrm{LH}$ receptor (LHR) on the membrane of Leydig cells, which subsequently decreased LH-mediated testosterone synthesis and secretion from Leydig cells. Another study by Seethalakshmi et al (9) reported that CsA decreased testosterone levels by inhibiting the activity of 17 alpha-hydroxylase uncompetitively and $17 \beta$-hydroxydteroid dehydrogenase activity competitively during testosterone biosynthesis, and the primary site for CsA inhibition was interrupted cyclic adenosine monophosphate stimulation. Furthermore, this report indicated that decreased testosterone secretion induced by CsA in Leydig cells directly influenced development of the male reproductive system and maintenance 
of reproductive ability. Seethalakshmi et al (10) injected exogenous testosterone into CsA-treated rats to increase endogenous testosterone levels. It was observed that the elevated doses of exogenous testosterone significantly restored reproductive organ weight, serum levels of follicle-stimulating hormone (FSH) and increased the germ cell count, thus indicating that CsA-induced impairment of spermatogenesis may be partially prevented by the exogenous administration of testosterone (10). However, exogenous testosterone was found to be insufficient in repairing damaged testicular tissue, as testosterone secretion and spermatogenesis were still influenced by CsA, and high rates of apoptosis were observed in sperm and spermatogenic cells (10).

Wenshen Shengjing Decoction (WSSJD) consists of 15 herbal medicines, primarily including Cornu Cervi Nippon Parvum, Panax ginseng, Cynomorium songaricum, Cistanche deserticola, Radix Astragali, Epimedium brevicornum and Angelica sinensis (11). WSSJD has been demonstrated to alleviate cyclophosphamide-induced spermatogenic apoptosis (11). To further improve the therapeutic effect of WSSJD, the present study developed a new WSSJD based on the mechanism of CsA-induced testicular tissue damage by adjusting the dosage of the WSSJD components. Modern pharmacological studies have demonstrated that antler velvet in WSSJD may function as a sex hormone (12); Radix Astragali may improve the proliferation and nutrient supply of testicular sertoli cells (13); and Panax ginseng may decrease testicular hyperoxide levels and inhibit spermatogenic apoptosis (14). The above-mentioned medicines may also increase kidney function and boost the development of spermatogenic cells (12-14). Clomifene citrate (CC) has been demonstrated to exert therapeutics effect on male infertility in previous clinical studies $(15,16)$. Furthermore, $\mathrm{CC}$ has been associated with improved testosterone and semen parameters, and the protective mechanisms of CC may be similar to those of WSSJD $(15,16)$. In addition, in a study by Wang et al $(17)$, $\mathrm{CC}$ was used as a treatment control to investigate the effect of the Chinese medicine Shengjing on spermatogenesis disturbances in mice. Based on this, CC was used as a positive control in the present study.

In the present study, the mechanism underlying the protective effect of new WSSJD in testicular tissues was investigated to determine the therapeutic efficacy of the modified medicine. Elucidating the protective mechanism may provide a basis for the use of new WSSJD in ameliorating CsA-induced spermatogenic damage.

\section{Materials and methods}

Animals. A total of 90 male Kunming mice (8-weeks-old; $35-40 \mathrm{~g}$ ) were provided by the Changchun Institute of Biological Products Co., Ltd. (Changchun, China). The present study was conducted with approval from the Ethics Committee of Jilin Medical University (Changchun, China). Mice were housed at $21 \pm 3^{\circ} \mathrm{C}$ and $55-65 \%$ relative humidity under a $12 \mathrm{~h}$ dark-light cycle. Mice were given free access to a standard laboratory mouse diet and sterile water.

Preparation of WSSJD. The 15 Chinese medicine components of WSSJD, including Cornu Cervi Nippon Parvum,
Panax ginseng, Cynomorium songaricum, Cistanche deserticola, Radix Astragali, Epimedium brevicornum and Angelica sinensis (11), were purchased from Tongrentang (Beijing, China). These materials were decocted according to the traditional method of Chinese medicinal decoction (18). In brief, the ingredients were weighed according to the recipe, and a total of $250 \mathrm{ml}$ distilled water was added to submerge the herbs. Herbs were soaked for $30 \mathrm{~min}$ and subsequently heated. The herbs were first boiled for $10 \mathrm{~min}$ and then the heat was reduced to a simmer for $20 \mathrm{~min}$. Following $30 \mathrm{~min}$ of heating, the liquid decoction was separated from the herbs, which were further decocted with $200 \mathrm{ml}$ distilled water at $80^{\circ} \mathrm{C}$ for another $30 \mathrm{~min}$. This process was repeated and the final volume of decoction liquid was filtered through 4-5 layers of gauze. Liquid was also squeezed from the herbs into the filtered decoction. The decoction was subsequently heated at $80^{\circ} \mathrm{C}$ in a water bath for $6-7 \mathrm{~h}$ until the concentration reached $2 \mathrm{~g}$ crude $\mathrm{drug} / \mathrm{ml}$, and the decoction was stored at $4^{\circ} \mathrm{C}$ prior to use. new WSSJD was prepared based on the formulation of WSSJD with minor adjustments to the dosages of Panax ginseng, Cynomorium songaricum, Radix Astragali and Epimedium brevicornum. The decocting method was the same as that of WSSJD. new WSSJD comprised of the following: Panax ginseng, $6 \mathrm{~g}$; Cynomorium songaricum, 9 g; Radix Astragali, 12 g; Epimedium brevicornum, 6 g; Cornu Cervi Nippon Parvum, $1 \mathrm{~g}$; Cistanche deserticola, 9 g; Angelica sinensis, 6 g; Flatstem Milkvetch Seed, 9 g; Rhizoma Dioscoreae, 15 g; Largehead Atractylodes Rhizome, 6 g; Ligusticum wallichii, 3 g; Radix Paeoniae Alba, $6 \mathrm{~g}$; Cinnamomum cassia, $1 \mathrm{~g}$; Costustoot, $1.5 \mathrm{~g}$ and Fructus Foeniculi, $3 \mathrm{~g}$.

The concentrations of crude drug extracts were determined as follows: Weight of the crude material/final volume. This calculation method is widely used in the study of traditional Chinese medicine (19-21).

Drug administration. Mice were randomly divided into 6 groups (15 mice per group) and were administrated with medicine intragastrically. The groups were as follows: Control (normal saline); dimethylsulfoxide (DMSO); CsA; CC; WSSJD; and new WSSJD. Mice in the CsA, CC, WSSJD and new WSSJD groups were intraperitoneally (i.p.) injected with $15 \mathrm{mg} / \mathrm{kg} / \mathrm{day}$ CsA (Shanxi Powerdone Pharmaceutics Co., Ltd., Datong, China) for 30 days, as described previously (8). Mice in the control and DMSO groups underwent a daily i.p. injection with an equal volume of normal saline or DMSO solvent, respectively, throughout the 30-day experimental period. The concentration of DMSO diluted in normal saline was $3.25 \%(\mathrm{v} / \mathrm{v})$. The CC group was administered with $21.6 \mathrm{mg} / \mathrm{kg} / \mathrm{day}$ CC (GKH Pharmaceutical, Ltd., Guangzhou, China) as described previously (13), which was also diluted in $3.25 \%(\mathrm{v} / \mathrm{v}) \mathrm{DMSO}(\mathrm{pH}=7.2)$. Mice in the WSSJD and new WSSJD groups were administered with $12 \mathrm{~g}$ crude drug $/ \mathrm{kg} / \mathrm{day}$ of WSSJD and new WSSJD, respectively.

Mouse euthanasia. At the end of the experimental period (at day 31), mice were placed in sealed cages, which were subsequently infused with $10-30 \% \mathrm{CO}_{2}$. When mice ceased to move for $5 \mathrm{~min}$, it was confirmed that mice had succumbed to $\mathrm{CO}_{2}$ exposure. 
Hematoxylin and eosin $(H \& E)$ staining. Mice testes were harvested and immediately fixed in $4 \%$ paraformaldehyde $(\mathrm{pH}=7.2)$ at room temperature for $24 \mathrm{~h}$, followed by ethanol dehydration, xylene treatment to remove the ethanol, wax embedding and sectioning. Histological sections $(5 \mu \mathrm{m})$ were obtained using a rotary microtome. These were affixed to glass slides for H\&E staining. Testicular sections were observed using highlight histopathological microscopy to evaluate the development of seminiferous tubules and obtain histometric data. The development of seminiferous tubules was assessed using the Johnsen scoring system (22).

ELISA. On day 30 of treatment, the mice were anesthetized with $10 \%$ chloral hydrate (Shanghai Guoyao Chemical Reagent Co., Ltd) at $0.004 \mathrm{ml} / \mathrm{g}$ of body weight i.p. prior to euthanasia with $\mathrm{CO}_{2}$. Trunk blood was harvested and the serum was separated and stored at $-20^{\circ} \mathrm{C}$. Briefly, blood was collected into $1.5-\mathrm{ml}$ tubes and stored at $4^{\circ} \mathrm{C}$ overnight. The tube was then centrifuged at $1,300 \mathrm{x}$ at $4^{\circ} \mathrm{C}$ for 6 min and the serum was collected. ELISA kits (Shanghai Elisa Biotech Co., Ltd., Shanghai, China) were used to determine the serum contents of testosterone (cat. no. EIA-2380) and LH (cat. no. EIA-2385) according to the manufacturer's protocol. The optical density (OD) was determined using a Model 680 microplate reader (Bio-Rad Laboratories, Inc., Hercules, CA, USA), and the levels of testosterone and $\mathrm{LH}$ were determined based on the standard curve.

Immunohistochemistry. Testicular tissues were fixed in $4 \%$ formaldehyde at room temperature for $24 \mathrm{~h}$ and embedded in paraffin. Paraffin sections were cut into $5 \mu \mathrm{m}$ sections and were subsequently dewaxed. Antigen retrieval was achieved by incubating sections in $0.01 \mathrm{M}$ citrate buffer $(\mathrm{pH}=6.0)$ at $95-98^{\circ} \mathrm{C}$ for $5 \mathrm{~min}$. The sections were subsequently blocked with $5 \%$ bovine serum albumin (Sigma-Aldrich; Merck KGaA, Darmstadt, Germany; A3675) at room temperature for $1 \mathrm{~h}$. Following blocking, sections were incubated with rabbit polyclonal antibodies against LHR (cat. no. L6792; Sigma-Aldrich; Merck KGaA; 1:200) or P450 side chain cleavage (P450scc; cat. no. ab75497; Abcam, Cambridge, UK; 1:200) at $4^{\circ} \mathrm{C}$ overnight in the dark. The streptavidin-biotin complex (SABC) method was used to detect the expression of LHR and P450scc using an SABC kit (SA2010; Boster Biological Technology, Pleasanton, CA, USA), according to the manufacturer's protocol. For the tissues isolated from negative control mice, the primary antibody was replaced with PBS. Leydig cells with yellow or brown staining on the membrane or plasma were considered to be LHR-positive cells. Five slides were obtained from each sample and five fields in the Leydig tissue areas were selected at random and assessed under a fluorescence microscope (magnification, $\mathrm{x} 400)$. The mean OD of positive cells in the Leydig tissue areas was obtained using Image Pro Plus 6.0 software (Media Cybernetics, Inc., Rockville, MD, USA). The expressions of LHR and P450scc were proportional to the OD values, with higher OD values representing higher protein expressions.

Terminal dexynucleotidyl transferase (TdT)-mediated dUTP nick-end labeling (TUNEL) assay. Mice testes were dissected, embedded in wax and sectioned. The sections (5- $\mu \mathrm{m}$ thick) were then stained using the TUNEL assay kit (MK1024;
Boster Biological Technology), according to the manufacturer's protocol. In brief, testicular tissues form control (normal saline), DMSO, CsA, CC, WSSJD and new WSSJD groups were stained using a 1:100 dilution of biotin labeled digoxin antibody for $30 \mathrm{~min}$ at $37^{\circ} \mathrm{C}$. Stained rat interstitial epithelial tissue (provided in the kit) was used as a positive control, and samples incubated with PBS instead of biotin labeled digoxin antibody were used as a negative control. The frequency of TUNEL-positive cells exhibiting green nuclear staining was evaluated using a laser scanning confocal microscope. A total of 10 random fields were assessed under high-magnification (x400) and positive cells were counted. The mean number of positive cells per field was calculated.

Propidium iodide (PI) or Annexin V-fluorescein isothiocyanate (FITC) staining and flow cytometry analysis. The cauda epididymidis was harvested following sacrifice and the epidermis was cut with a blade to release the sperm from the epididymis into $2 \mathrm{ml} \mathrm{PBS}$ at $37^{\circ} \mathrm{C}$ to obtain epididymis suspensions. The suspensions were subsequently incubated at $37^{\circ} \mathrm{C}$ for $10 \mathrm{~min}$ to allow sperm to swim out. Aggregations of sperm were discarded and the remaining sperm samples were isolated and resuspended in PBS to give a concentration of $10^{6}$ cells $/ \mathrm{ml}$. Sperm apoptosis was analyzed using a Annexin V-FITC Apoptosis Detection kit (Nanjing KeyGen Biotech Co., Ltd., Nanjing, China), according to the manufacturer's protocol. In brief, $1 \mathrm{ml}$ of sperm suspension was stained with $10 \mu \mathrm{l}$ PI or with $500 \mu \mathrm{l}$ binding buffer and $5 \mu \mathrm{l}$ Annexin V-FITC in the dark for $10 \mathrm{~min}$ at room temperature. Cells were immediately analyzed by Epies XL flow cytometry (Beckman Coulter, Inc.) and using a TetraONE ${ }^{\mathrm{TM}}$ System (6915050; Beckman Coulter, Inc.). Cells in the early stages of apoptosis were identified by Annexin V-positive staining and necrotic cells were identified by PI-positive staining.

Statistical analysis. Data analysis was performed using SPSS 13.0 software (SPSS, Inc., Chicago, IL, USA) and expressed as the mean + or \pm standard deviation as indicated. Differences between groups were evaluated for significance using one-way analysis of variance followed by a Tukey's post hoc test. $\mathrm{P}<0.05$ was considered to indicate a statistically significant difference.

\section{Results}

Effects of new WSSJD on the development of testicular seminiferous tubules. To investigate the potential protective effect of new WSSJD on the development of mouse testicular seminiferous tubules, the morphology of H\&E-stained mouse testes were compared under light microscopy. The seminiferous tubules of mice from the CsA and CC groups exhibited shrinkage of the tubule fringe, decreased tubule diameters and reduced layers of testicular seminiferous epithelium compared with the control. In addition, spermatogenic cells exhibited a disordered arrangement, the number of spermatogenic cells was reduced and few mature sperm were visible in the lumen (Fig. 1). In mice from the DMSO, WSSJD and new WSSJD groups, more layers of testicular seminiferous epithelia were visible and the seminiferous tubule fringe was integrated without shrinkage or collapse (Fig. 1). The Johnsen scores did not differ significantly between the DMSO group and control 
groups, or between the CC and DMSO groups. However, the Johnsen score was significantly decreased in the CsA group and WSSJD group compared with the control group, and the scores of the WSSJD and new WSSJD groups were significantly increased compared with the CC or CsA group $(\mathrm{P}<0.05$; Table I). These results indicated that new WSSJD and WSSJD promoted the development of seminiferous epithelium following CsA treatment.

Effects of new WSSJD on serum testosterone and LH. The levels of testosterone and $\mathrm{LH}$ in the serum were subsequently measured. Serum levels of testosterone and LH were unaffected following DMSO administration. By contrast, serum testosterone was significantly downregulated and LH was significantly upregulated in CsA-treated mice, relative to controls $(\mathrm{P}<0.05$; Fig. 2$)$, and new WSSJD administration significantly restored testosterone to near control levels $(\mathrm{P}<0.05$; Fig. 2). For serum LH, the protective effects of WSSJD was similar to that of CC; however, new WSSJD decreased serum testosterone to a significantly lower level than that observed with CC and WSSJD ( $\mathrm{P}<0.05$; Fig. 2), suggesting a superior protective effect of new WSSJD over CC or WSSJD.

Effects of new WSSJD on the expressions of LHR and P450scc. The expressions of P450scc and LHR were assessed in testicular Leydig cells by immunohistochemistry (Figs. 3 and 4). In the control group, LHR was expressed on the outer membranes of Leydig cells between the seminiferous tubules (Fig. 3A), and P450cc was expressed in the cytoplasm of testicular Leydig cells (Fig. 4A). DMSO treatment had no significant effect on the expressions of LHR and P450scc. By contrast, CsA treatment significantly decreased the expressions of LHR and P450scc compared with control mice $(\mathrm{P}<0.05$; Figs. 3B and 4B). In turn, the expressions of LHR and P450scc were significantly increased by treatment with CC, WSSJD or new WSSJD compared with the CsA group $(\mathrm{P}<0.05$; Figs. $3 \mathrm{~B}$ and 4B). In addition, levels of LHR and P450scc in testicular Leydig cells were significantly higher in WSSJD and new WSSJD mice compared with $\mathrm{CC}$ mice $(\mathrm{P}<0.05$; Figs. $3 \mathrm{~B}$ and $4 \mathrm{~B})$, and new WSSJD induced a significantly greater upregulation than WSSJD $(\mathrm{P}<0.05$; Figs. 3B and 4B).

Effect of new WSSJD on spermatogenic cell apoptosis. The apoptosis of spermatogenic cells in the mouse testes was analyzed by a TUNEL assay. The nuclei of apoptotic cells were principally observed in the spermatogonia and primary spermatocytes (Fig. 5A). Spermatogonia are larger than spermatocytes and are located closer to the basement membrane (23). DMSO treatment had no significant effect on the number of apoptotic spermatogenic cells in the testes. By contrast, treatment with CsA significantly increased the number of apoptotic spermatogenic cells compared with the control and DMSO groups $(\mathrm{P}<0.05$; Fig. 5B). In turn, administration of CC, WSSJD or new WSSJD significantly reduced $\mathrm{Cs} A-i n d u c e d$ apoptosis $(\mathrm{P}<0.05$; Fig. 5B). Furthermore, the number of apoptotic testicular spermatogenic cells in the WSSJD and new WSSJD groups was significantly reduced compared with the CC group $(\mathrm{P}<0.05)$, and new WSSJD was significantly more effective than WSSJD $(\mathrm{P}<0.05$; Fig. 5B).
Table I. Effect of new WSSJD on Johnsen scoring of the testes.

\begin{tabular}{lc}
\hline Groups & Johnsen score $(\leq 10)$ \\
\hline Control & $9.75 \pm 0.25$ \\
DMSO & $9.50 \pm 0.25$ \\
CsA & $7.25 \pm 0.43^{\mathrm{a}}$ \\
CC & $7.33 \pm 0.29^{\mathrm{a}}$ \\
WSSJD & $9.00 \pm 0.25^{\mathrm{a}-\mathrm{c}}$ \\
New WSSJD & $9.25 \pm 0.25^{\mathrm{b}, \mathrm{c}}$
\end{tabular}

Data are expressed as the mean \pm standard deviation. ${ }^{\text {aP }}<0.05$ vs. control; ${ }^{\mathrm{b}} \mathrm{P}<0.05$ vs. Cs A group; ${ }^{\mathrm{C}} \mathrm{P}<0.05$ vs. CC group. DMSO, dimethylsulfoxide; CsA, cyclosporine A; CC, clomifene citrate; WSSJD, Wenshen Shengjing Decoction.

Effects of new WSSJD on the survival and early apoptosis of sperm. To verify the protective effects of new WSSJD against CsA-induced sperm apoptosis, the survival and early apoptosis of sperm in the epididymis were determined. Epididymal sperm were stained with PI or Annexin V and analyzed by flow cytometry (Figs. 6 and 7). In accordance with the aforementioned results, CsA treatment significantly reduced the percentage of live sperm and significantly increased the percentage of early apoptotic sperm $(\mathrm{P}<0.05$; Figs. $6 \mathrm{~B}$ and 7B), while DMSO treatment had no effect. WSSJD and new WSSJD significantly increased the percentage of live sperm $(\mathrm{P}<0.05$; Fig. 6B) and reduced the percentage of early apoptosis sperm $(\mathrm{P}<0.05$; Fig. 7B) compared with CsA treatment. The percentages of live and early apoptotic sperm in WSSJD and CC mice did not differ significantly, while new WSSJD induced a significantly greater increase in live sperm percentage compared with the CC group $(\mathrm{P}<0.05$; Fig. 6B) and significantly decreased the percentage of early apoptotic sperm compared with the CC and WSSJD groups ( $\mathrm{P}<0.05$; Fig. 7B).

\section{Discussion}

It has previously been documented that long-term use of CsA as an immunosuppressive agent affects reproductive capacity (6). $\mathrm{Xu}$ (24) reported that the morphology and vitality of sperm in patients treated with CsA were significantly lower than that in an untreated group, as observed in the semen samples of 26 renal transplant recipients treated with various doses of CsA and 12 healthy volunteers. It was also observed that head deformity rates of sperm were significantly higher in CsA-treated recipients (24). These results suggested that CsA had a dose-dependent effect on semen parameters. Therefore, studies are warranted to identify novel pharmacological agents capable of alleviating CsA-induced testicular damage and improving male reproductive ability following organ transplantation.

The specific microenvironment of the testis promotes spermatogenesis, and thus impairment of testicular structure and function may lead to spermatogenic arrest (25). Monteiro et al (26) treated Wistar rats with CsA at a dose of $15 \mathrm{mg} / \mathrm{kg}$ per day for 56 days, and observed an increased volumetric proportion of connective tissue and decreased volumetric proportion of Leydig cells in CsA-treated rats. It was also observed that CsA caused 


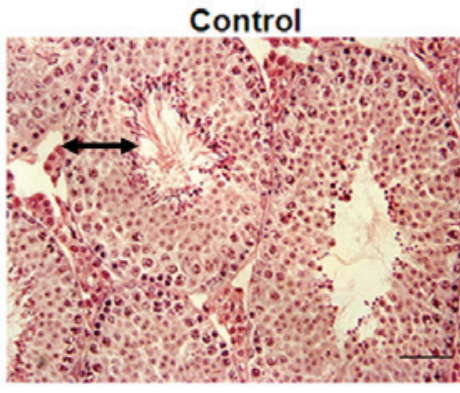

CC

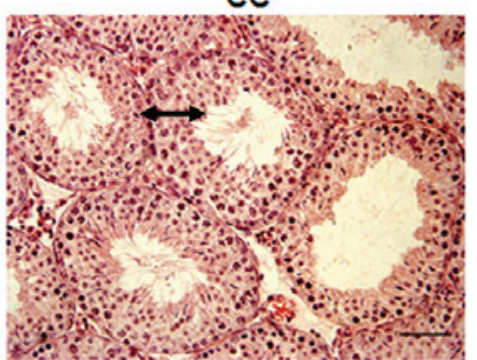

DMSO

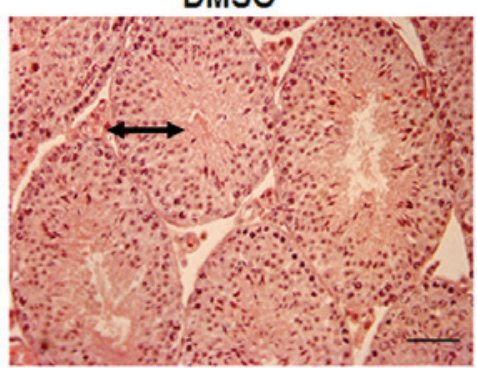

WSSJD

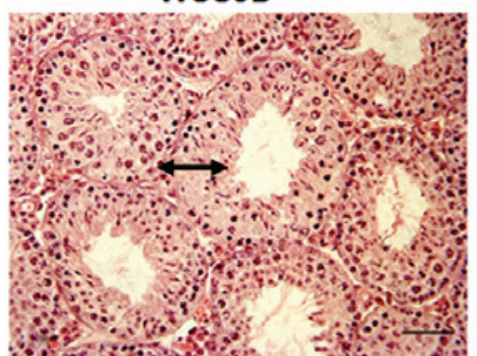

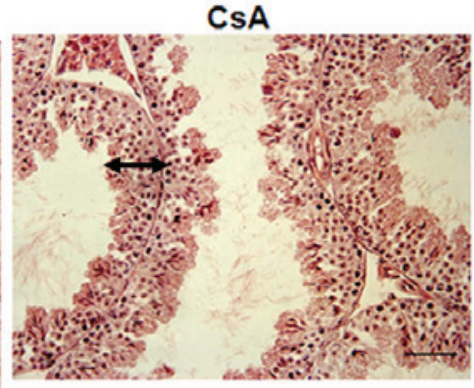

New WSSJD

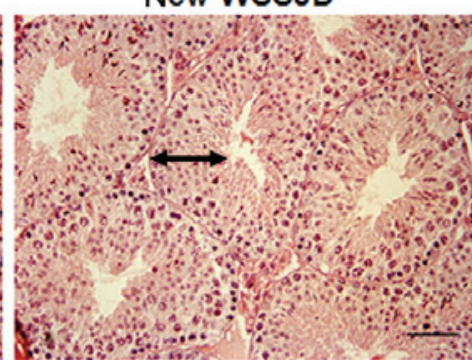

Figure 1. Hematoxylin and eosin staining of mouse testicular tissue. Mice were administered with CsA, followed by CC, WSSJD, new WSSJD or DMSO. Untreated mice were used as a negative control. The testes were harvested 30 days later and stained with hematoxylin and eosin. CsA, cyclosporine A; CC, clomifene citrate; WSSJD, Wenshen Shengjing Decoction; DMSO, dimethylsulfoxide. The length of arrows represented the thickness of spermatogenic tubule, and the ratio of their thickness was 1.4:1.4:1.2:1.22:1.3:1.4 in control, DMSO, CsA, WSSJD and new WSSJD group, respectively. Magnification, x200; Scale bar, $100 \mu \mathrm{m}$.

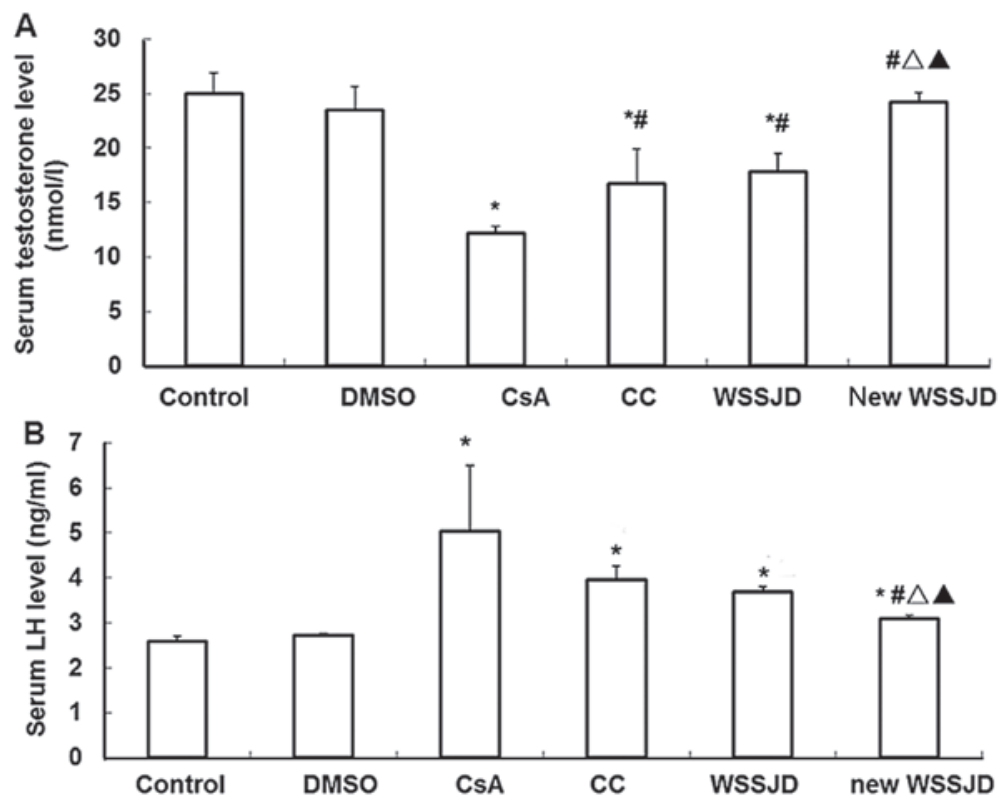

Figure 2. ELISA analysis of serum testosterone and LH. Mice were administered with CsA and treated with CC, WSSJD, new WSSJD or DMSO. Serum was harvested 30 days later. Levels of (A) testosterone and (B) LH in the serum were determined by ELISA analysis. Untreated mice were used as a negative control. " $\mathrm{P}<0.05$ vs. control; ${ }^{\#} \mathrm{P}<0.05$ vs. CsA group; ${ }^{\Delta} \mathrm{P}<0.05$ vs. CC group; ${ }^{\wedge} \mathrm{P}<0.05$ vs. WSSJD group. LH, luteinizing hormone; CsA, cyclosporine A; CC, clomifene citrate; WSSJD, Wenshen Shengjing Decoction; DMSO, dimethylsulfoxide. Data are presented as the mean \pm standard deviation.

seminiferous epithelium degeneration, resulting in Sertoli cell vacuolization, abnormal round and elongated spermatids and the accumulation of residual cytoplasm at the epithelium border adjacent to the lumen (26). In the present study, Kunming mice were treated with $15 \mathrm{mg} / \mathrm{kg}$ CsA daily for 30 days, which lead to shrinkage and decreased diameters of the seminiferous tubules, reduction of the seminiferous epithelium layers, disordered arrangement of the seminiferous cells, a decrease in mature sperm in the lumen and a significantly decreased Johnsen score.
In addition, treatment with CsA treatment severely damaged the testicular structure. The present study also evaluated the protective effects of new WSSJD, as a novel Chinese medicine, on the testes of CsA-treated mice. It was observed that the testicular seminiferous epithelia layers and arrangement of the seminiferous cells were restored following treatment with new WSSJD. In addition, mice in the new WSSJD group exhibited integrated seminiferous tubules without shrinkage or collapse, and had a significantly higher Johnsen score compared with CsA-treated 


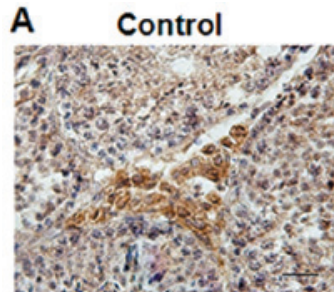

WSSJD

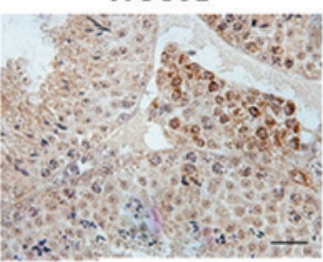

DMSO

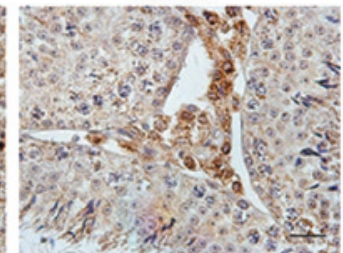

New WSSJD

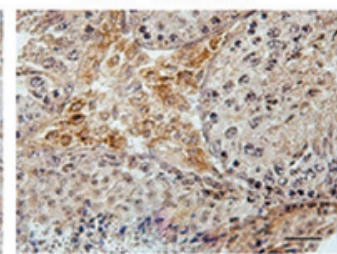

CsA

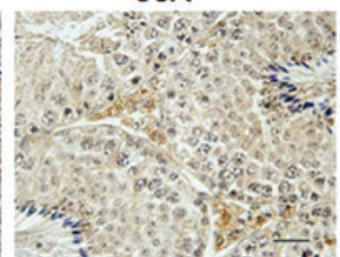

Negative

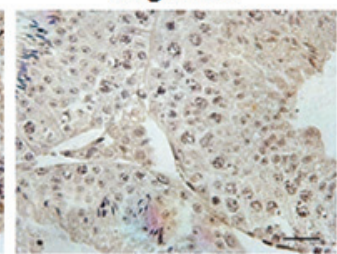

CC

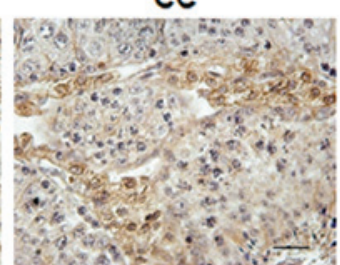

B

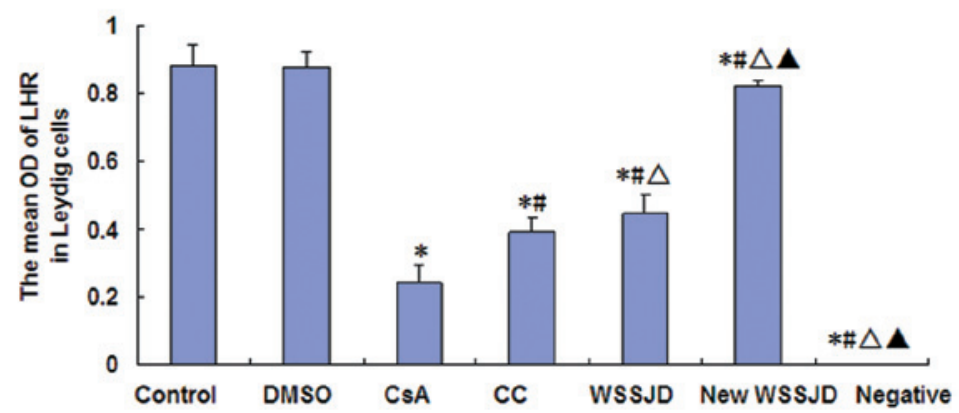

Figure 3. Immunohistochemistry of LHR protein in Leydig cells. (A) Immunohistochemistry identified an accumulation of Leydig cells positive for LHR (brown) between the seminiferous tubules. Negative tissues were treated using PBS instead of antibodies against LHR. Magnification, x400; Scale bar, $50 \mu \mathrm{m}$. (B) The mean OD of LHR-positive cells in each group. " $\mathrm{P}<0.05$ vs. control; ${ }^{\sharp} \mathrm{P}<0.05$ vs. CsA group; ${ }^{\triangle} \mathrm{P}<0.05$ vs. CC group; ${ }^{\wedge} \mathrm{P}<0.05$ vs. WSSJD group. LHR, luteinizing hormone receptor; OD, optical density; CsA, cyclosporine A; CC, clomifene citrate; WSSJD, Wenshen Shengjing Decoction; DMSO, dimethylsulfoxide. Data are presented as the mean \pm standard deviation.

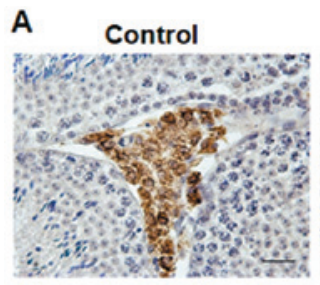

DMSO

CsA

cC

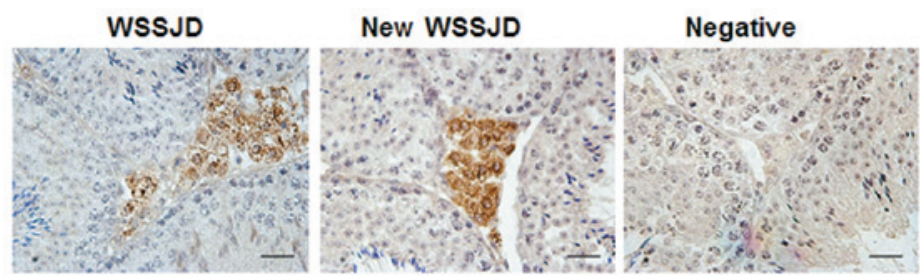

B

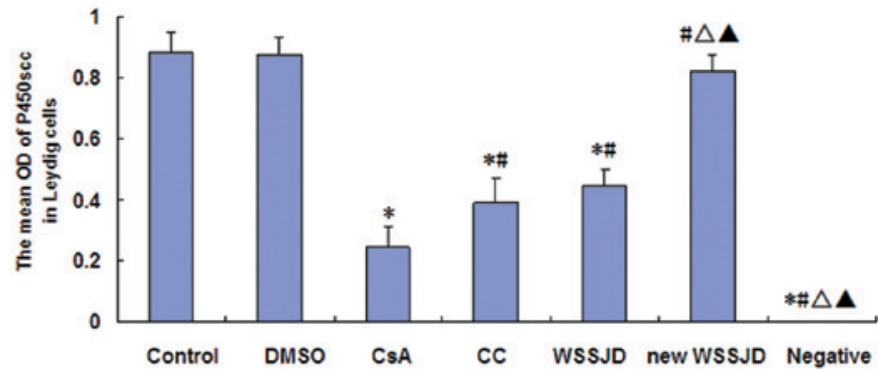

Figure 4. Immunohistochemistry of P450scc protein in Leydig cells. (A) Immunohistochemistry identified positive staining for p450scc (brown) in the cytoplasm of Leydig cells. Negative sections were treated with PBS instead of antibodies against P450scc. Magnification, $\mathrm{x} 400$; Scale bar, $50 \mu \mathrm{m}$. (B) The mean OD of $\mathrm{P} 450$ cc-positive cells in each group. ${ }^{*} \mathrm{P}<0.05$ vs. control; ${ }^{\#} \mathrm{P}<0.05$ vs. CsA group; ${ }^{\wedge} \mathrm{P}<0.05$ vs. CC group; ${ }^{\Delta} \mathrm{P}<0.05$ vs. WSSJD group. OD, optical density; P450scc, P450 side chain cleavage; CsA, cyclosporine A; CC, clomifene citrate; WSSJD, Wenshen Shengjing Decoction; DMSO, dimethylsulfoxide. Data are presented as the mean \pm standard deviation. 
A

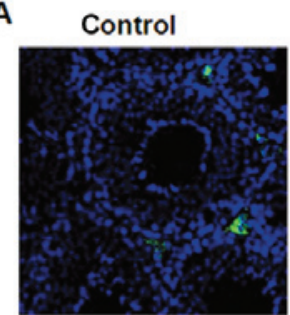

WSSJD

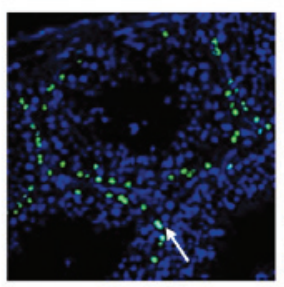

DMSO

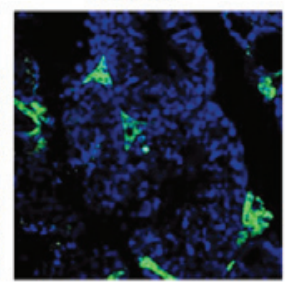

New WSSJD

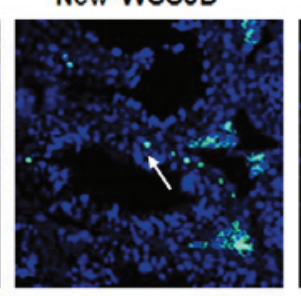

CsA

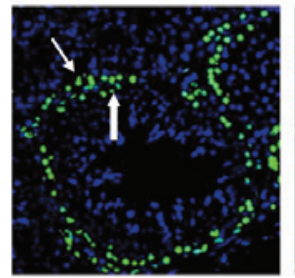

Positive

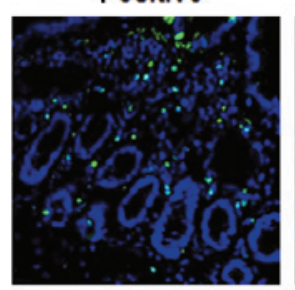

cc

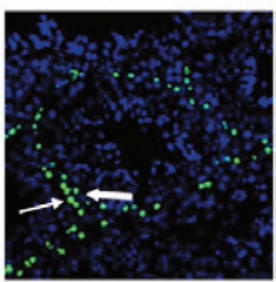

Negative

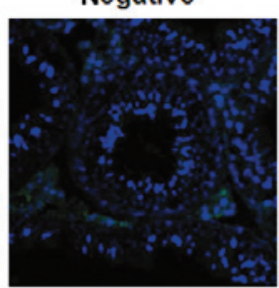

B

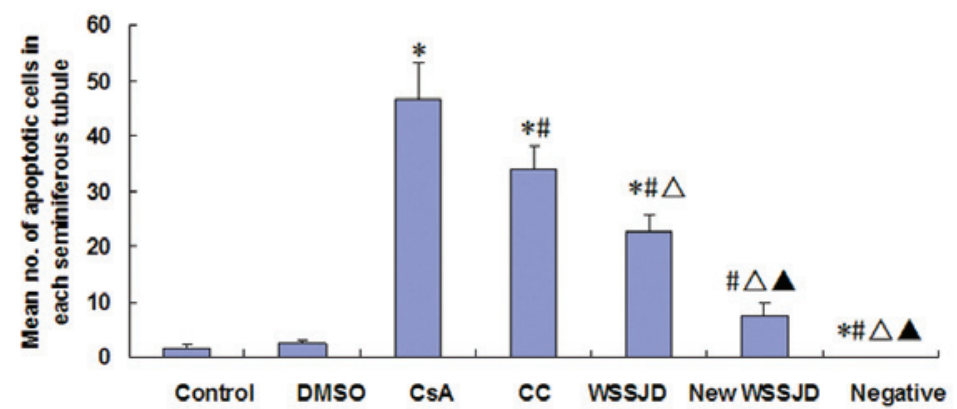

Figure 5. TUNEL staining of mouse testes. (A) TUNEL staining (green) and Hoechst 33342 immunostaining (blue) of sections derived from the testes of mice administered with CsA followed by CC, WSSJD, new WSSJD or DMSO. Untreated mice were used as a control. Spermatogoniums and primary spermatocytes were observed. Fine arrows indicate spermatogonia, which were close to the basal membrane of spermatogenic tubules. Thick arrows indicate primary spermatocytes, which were close to spermatogonia/inside spermatogonia. Interstitial tissue of the testis provided by the TUNEL kit were used as positive sections. Negative sections were treated with PBS instead of biotin-labeled digoxin antibody. Magnification, x400. (B) Quantification of apoptosis in spermatogenic cells. ${ }^{*} \mathrm{P}<0.05$ vs. control; ${ }^{\#} \mathrm{P}<0.05$ vs. CsA group; ${ }^{\Delta} \mathrm{P}<0.05$ vs. CC group; ${ }^{\wedge} \mathrm{P}<0.05$ vs. WSSJD group. CsA, cyclosporine A; CC, clomifene citrate; WSSJD, Wenshen Shengjing Decoction; DMSO, dimethylsulfoxide. Data are presented as the mean \pm standard deviation.

mice. These results suggested that WSSJD significantly repaired CsA-induced testicular damage, indicating that this herbal medicine compound may be an effective treatment in the prevention of CsA-induced testicular damage. Whether or not this cell repair is dependent on the niche within the epithelium is also an important question, which should be investigated in future studies.

The hypothalamic-pituitary-testicular axis serves an important role in the regulation of genital activity (27). The development and functionality of the male genital organs are regulated by hormones from the hypothalamus and pituitary glands (28). Krueger et al (29) treated Sprague Dawley rats with $25 \mathrm{mg} / \mathrm{kg} /$ day CsA or $40 \mathrm{mg} / \mathrm{kg} /$ day CsA for 6 days and observed that serum levels of LH and FSH increased by 2-4 fold, while P450scc expression decreased to $30 \%$ of that in the control group. In addition, serum testosterone levels were significantly decreased in CsA-treated mice, resulting in impaired spermatogenesis (29). In the present study, it was demonstrated that CsA treatment significantly decreased the expression of LHR in Leydig cells. Although serum LH was increased, decreased expression of LHR may have impaired LH-mediated recovery of testosterone biosynthesis and decreased the expression of P450scc, as the rate-limiting enzyme of testosterone biosynthesis (30), thus affecting testosterone biosynthesis by Leydig cells. The novel ingredients in new WSSJD, such as pilose antler, exhibit effects similar to sex hormones (12), which may improve serum testosterone levels and decrease serum LH levels, thus increasing LHR and p450scc expression in Leydig cells. Notably, the present results indicated that new WSSJD stimulated testosterone biosynthesis and secretion in Leydig cells to promote spermatogenesis.

CsA-induced oxidative stress and testis damage induce the dysplasia of sperm and spermatogenic cells (31). It has been reported that long-term CsA treatment damages the antioxidant system in animal testicular tissues, leading to decreased levels of glutathione, glutathione peroxidase and hydrogen peroxide levels and increased levels of malonic dialdehyde in the testes (31). Therefore, excessive levels of reactive oxygen species in the testicular tissues cannot be eliminated, leading to the peroxidation of sperm membrane lipids, DNA damage and decreased sperm vitality. In the present study, it was observed that CsA treatment significantly increased DNA breakage and the apoptotic rate of spermatogenic cells in seminiferous tubules. The survival rate of sperm in the epididymis also decreased, while the percentage of early apoptotic sperm was increased, indicating that the spermatogenic activity of the testes was significantly damaged. Türk et al (32) documented that the protective effect of ellagic acid on CsA-induced testicular damage was associated with oxidative stress in male rats. The new WSSJD used in the present study contains various antioxidant components, including the herbal medicine Ginseng, which has previously been demonstrated to significantly decrease levels of hyperoxide in the testes (14). 

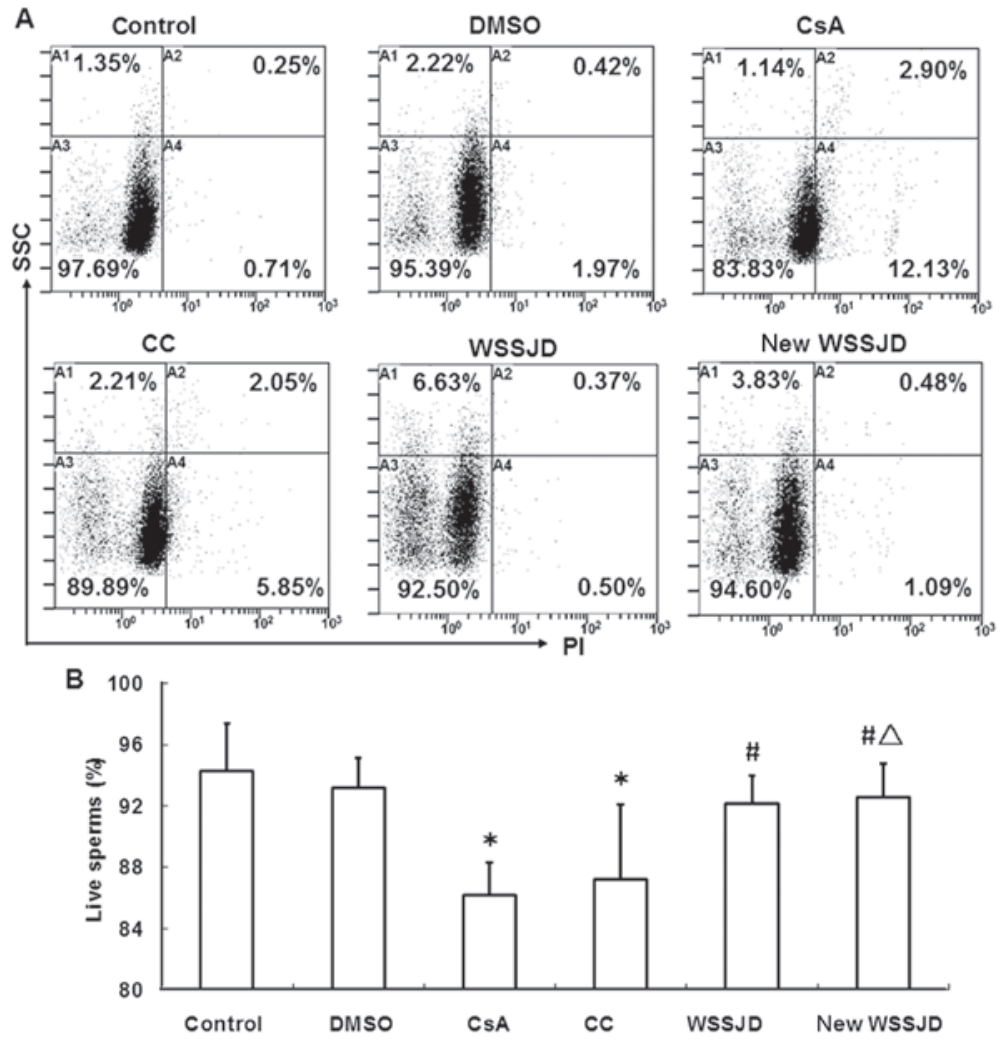

Figure 6. Flow cytometry analysis of live sperm. Mice were administered with CsA followed by CC, WSSJD, new WSSJD or DMSO. Untreated mice were used as a control. (A) Sperm were isolated from the epididymis, stained with propidium iodide and analyzed using flow cytometry. Percentages displayed in the lower left quadrant represent the PI-negative (live) cells. (B) Percentages of live sperm. ${ }^{*} \mathrm{P}<0.05$ vs. control; ${ }^{~} \mathrm{P}<0.05$ vs. CsA group; ${ }^{\wedge} \mathrm{P}<0.05$ vs. CC group; ${ }^{\wedge} \mathrm{P}<0.05$ vs. WSSJD group. CsA, cyclosporine A; CC, clomifene citrate; WSSJD, Wenshen Shengjing Decoction; DMSO, dimethylsulfoxide. Data are presented as the mean \pm standard deviation.
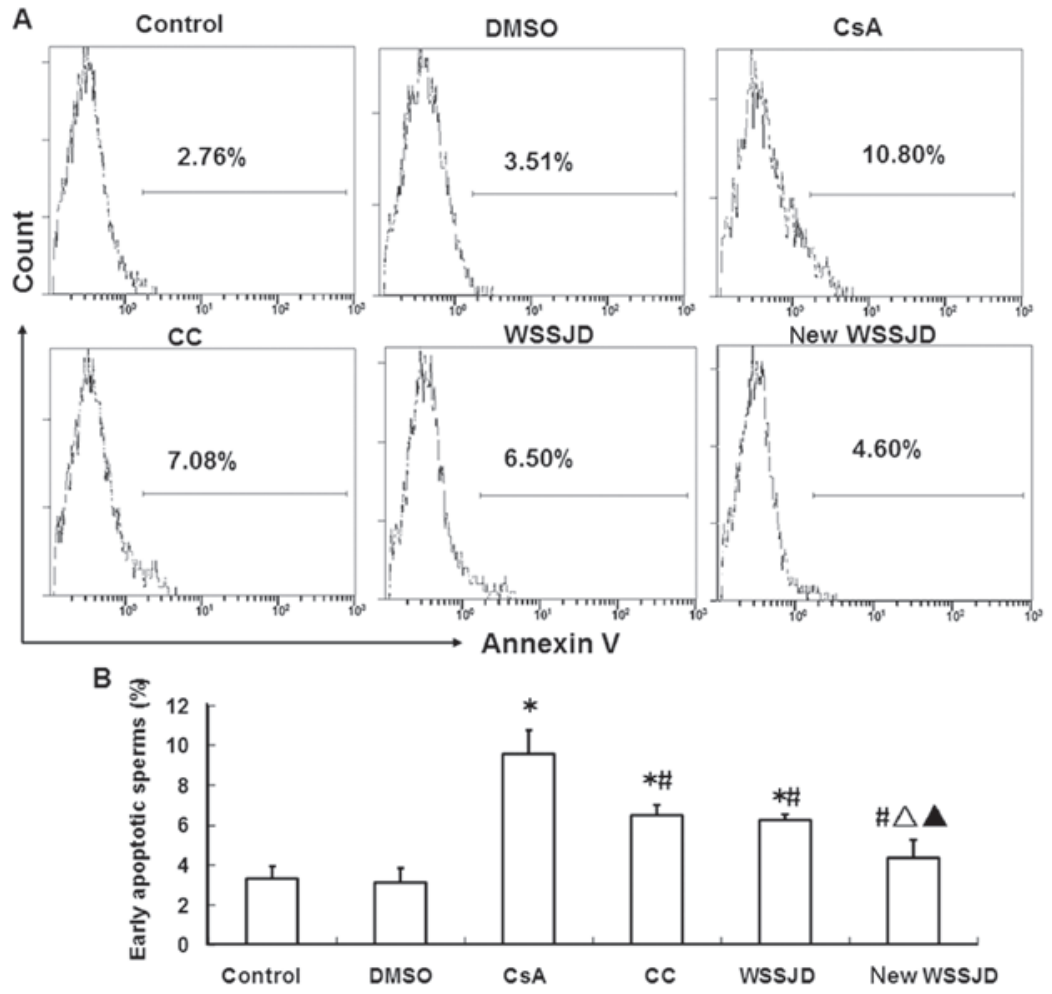

Figure 7. Flow cytometry analysis of early apoptotic sperm. Mice were administered with CsA followed by CC, WSSJD, new WSSJD or DMSO. Untreated mice were used as a control. (A) Sperm were isolated from the epididymis and stained using an Annexin V-fluorescein isothiocyanate Apoptosis Detection kit. Annexin V-positive staining was used to identify early apoptotic sperm. (B) Percentages of early apoptotic sperm. ${ }^{*} \mathrm{P}<0.05$ vs. control; ${ }^{\text {\#P }}<0.05$ vs. CsA group; ${ }^{\wedge} \mathrm{P}<0.05$ vs. CC group; ${ }^{\wedge} \mathrm{P}<0.05$ vs. WSSJD group. CsA, cyclosporine A; CC, clomifene citrate; WSSJD, Wenshen Shengjing Decoction; DMSO, dimethylsulfoxide. Data are presented as the mean \pm standard deviation. 
In the present study, the effects of new WSSJD on CsA-induced impairment of testosterone synthesis and spermatogenic apoptosis were investigated, and it was observed that new WSSJD significantly decreased the apoptotic rates of spermatogenic cells and sperm, and thus repaired damage to the testicular seminiferous epithelium. The morphology of testicular seminiferous tubules and the apoptosis of spermatogenic cells and sperm were investigated using histochemistry and flow cytometry. Although cell cycle distribution and daily sperm production were not investigated, they will be the focus of future studies by our group.

In conclusion, compared with traditional WSSJD, new WSSJD significantly increased testosterone levels in the testes and decreased the apoptosis of spermatogenic cells and sperm, which effectively repaired CsA-induced testicular damage. These results indicate that new WSSJD may be a useful pharmacological agent in the treatment and prevention of CsA-induced testicular damage.

\section{Acknowledgements}

The present study was supported by the Key Research Project of the Scientific and Technological Development Program of Jilin Province (grant no. 20140204033YY), the College Science and Technology Program of Shandong Province (grant no. J13LL04) and the Undergraduate Training Programs for Innovation and Entrepreneurship of Jilin Province (grant no. 2014024).

\section{References}

1. Pedersen $M$ and Seetharam A: Infections after orthotopic liver transplantation. J Clin Exp Hepatol 4: 347-360, 2014.

2. Vora GK and Ciolino JB: Corneal allograft reaction associated with nonocular inflammation. Digit J Ophthalmol 20: 29-31, 2014.

3. De Pasquale C, Veroux M, Indelicato L, Sinagra N, Giaquinta A, Fornaro M, Veroux P and Pistorio ML: Psychopathological aspects of kidney transplantation: Efficacy of a multidisciplinary team. World J Transplant 4: 267-275, 2014.

4. El-Gowelli HM and El-Mas MM: Central modulation of cyclosporine-induced hypertension. Naunyn Schmiedebergs Arch Pharmacol 388: 351-361, 2015.

5. Eid MM,Abdel-Hamid IA, Sobh MA and el-Saied MA: Assessment of sperm motion characteristics in infertile renal transplant recipients using computerized analysis. Int J Androl 19: 338-344, 1996.

6. Zahra A, Gholamreza N, Farokhi F and Shalizar Jalali A: Attenuation of cyclosporine-induced sperm impairment and embryotoxicity by crataegus monogyna fruit aqueous extract. Cell J 15: 198-205, 2013.

7. He Z, Qiu J, Li J, Zhao D, Chen G and Chen L: Long-term effects of conversion from cyclosporine to rapamycin on testicular function and morphology in a rat transplantation model. Transplant Proc 45: 763-769, 2013.

8. Ali RB, Klouz A, Boubaker S,Lakhal M and Belkahia C: An animal model of testicular toxicity by cyclosporine: Evaluation and protection. Fundam Clin Pharmacol 23: 241-246, 2009.

9. Seethalakshmi L, Flores C, Malhotra RK, Pallias JD, Tharakan D, Khauli RB and Menon M: The mechanism of cyclosporine's action in the inhibition of testosterone biosynthesis by rat Leydig cells in vitro. Transplantation 53: 190-195, 1992.

10. Seethalakshmi L,Flores C,Carboni AA and Menon M: Quantitative maintenance of spermatogenesis in cyclosporine-treated rats by exogenous administration of testosterone propionate. J Androl 11: 491-497, 1990.

11. Pan XY, Wang XY, Wang XN, Sun ZX, Wang D, Wang $X$, Yang YY and Li ZX: Effects of 'Wenshen Shengjing Decoction' on cyclophosphamide induced spermatogenic cell apoptosis and histone H3K9 dimethylation. Shanghai Zhong Yi Yao Za Zhi 48: 82-86, 2014 (In Chinese).
12. Xu ZH, Li SF, Wang JY, Zhou R and Tian SJ: Extraction of sex hormone from antler velvet with supercritical CO2. Zhongguo Zhong Yao Za Zhi 32: 2000-2003, 2007 (In Chinese).

13. Zhao LP, Xu Z, Zhang M, Sun HC and Tang F: Effects of Fructus Lycii and Radix astragali on the function of sertoli cells in rat testes. Zhonghua Nan Ke Xue 13: 82-86, 2007 (In Chinese).

14. Xu L, Liu XH and Zhang L: Effects of total ginsenosides on the $\mathrm{NO}, \mathrm{NOS}$ and total antioxygen capacity in the testis of mouse. Acta Academiae Medicinae CPAPF 5: 507-508, 2006 (In Chinese).

15. Patel DP, Brant WO, Myers JB, Presson AP, Johnstone EB, Dorais JA, Aston KI, Carrell DT and Hotaling JM: The safety and efficacy of clomiphene citrate in hypoandrogenic and subfertile men. Int J Impot Res 27: 221-224, 2015.

16. ElSheikh MG, Hosny MB, Elshenoufy A, Elghamrawi H, Fayad A and Abdelrahman S: Combination of vitamin $\mathrm{E}$ and clomiphene citrate in treating patients with idiopathic oligoasthenozoospermia: A prospective, randomized trial. Andrology 3: 864-867, 2015.

17. Wang R, Xu L, Zhang WX, Wu YF, Shi JH and Zhang YX: Shengjing Granule: An Effective Chinese Medicine for Spermatogenic Disturbance in Mice. Zhonghua Nan Ke Xue 14: 1046-1049, 2008 (In Chinese).

18. Fan BT: Chinese Medicine Pharmacy, Shanghai Science and Technology Press 12: 62-68, 1997 (In Chinese).

19. Zhang J, Li H, Lu L, Yan L, Yang X, Shi Z and Li D: The Yiqi and Yangyin Formula ameliorates injury to the hematopoietic system induced by total body irradiation. J Radiat Res 58: 1-7, 2017.

20. Minami M, Konishi T, Jiang Z, Arai T and Makino T: Effect of Shin'iseihaito on murine allergic reaction induced by nasal sensitization. J Tradit Complement Med 6: 252-256, 2015.

21. Rong R, Li RR, Hou YB, Li J, Ding JX, Zhang CB and Yang Y: Mahuang-xixin-fuzi decoction reduces the infection of influenza a virus in kidney-yang deficiency syndrome mice. J Ethnopharmacol 192: 217-224, 2016.

22. Johnsen SG: Testicular biopsy score count-a method for registration of spermatogenesis in human testes: Normal values and results in 335 hypogonadal males. Hormones 1: 2-25, 1970.

23. Mays-Hoopes LL, Bolen J, Riggs AD and Singer-Sam J: Preparation of spermatogonia, spermatocytes, and round spermatids for analysis of gene expression using fluorescence-activated cell sorting. Biol Reprod 53: 1003-1011, 1995.

24. Xu LG: Influence of cyclosporin A on the semen parameters of the patients after renal transplantation. J Clin Urol 20: 603-605, 2005.

25. Pintus E, Ros-Santaella JL and Garde JJ: Beyond testis size: Links between spermatogenesis and sperm traits in a seasonal breeding mammal. PLoS One 10: e0139240, 2015.

26. Monteiro JC, Predes FS, Matta SL and Dolder H: Heteropterys aphrodisiaca infusion reduces the collateral effects of cyclosporine A on the testis. Anat Rec (Hoboken) 291: 809-817, 2008.

27. Oseko F, Note S, Morikawa K, Endo J, Taniguchi A and Imura H: Influence of chronic hyperprolactinemia induced by sulpiride on the hypothalamo-pituitary-testicular axis in normal men. Fertil Steril 44: 106-111, 1985.

28. Wisniewski P, Romano RM, Kizys MM, Oliveira KC Kasamatsu T, Giannocco G, Chiamolera MI, Dias-da-Silva MR and Romano MA: Adult exposure to bisphenol A (BPA) in Wistar rats reduces sperm quality with disruption of the hypothalamic-pituitary-testicular axis. Toxicology 329: 1-9, 2015.

29. Krueger BA, Trakshel GM, Sluss PM and Maines MD: Cyclosporin-mediated depression of luteinizing hormone receptors and heme biosynthesis in rat testes: A possible mechanism for decrease in serum testosterone. Endocrinology 129: 2647-2654, 1991.

30. Nolan CJ and Payne AH: Genotype at the P450scc locus determines differences in the amount of P450scc protein and maximal testosterone production in mouse Leydig cells. Mol Endocrinol 4: 1459-1464, 1990.

31. Türk G, Ateşşahin A, Sönmez M, Yüce A and Ceribaşi AO: Lycopene protects against cyclosporine A-induced testicular toxicity in rats. Theriogenology 67: 778-785, 2007.

32. Türk G, Sönmez M, Ceribași AO, Yüce A and Ateşşahin A: Attenuation of cyclosporine A-induced testicular and spermatozoal damages associated with oxidative stress by ellagic acid. Int Immunopharmacol 10: 177-182, 2010.

This work is licensed under a Creative Commons Attribution-NonCommercial-NoDerivatives 4.0 International (CC BY-NC-ND 4.0) License. 